\title{
ELECTRON BACKSCATTER DIFRACTION: A POWERFUL TOOL FOR PHASE IDENTIFICATION IN THE SEM
}

J. R. MICHAEL, R. P. GOEHNER
Sandia National Laboratories, Albuquerque, NM 87185-1405, jrmicha@sandia.gov $4: 08$

ABSTRACT

EBSD in the SEM has been developed into a tool that can provide identification of unknown crystalline phases with a spatial resolution that is better than one micrometer. This technique has been applied to a wide range of materials. Use of the HOLZ rings in the EBSD patterns has enabled the reduced unit cell to be determined from unindexed EBSD patterns. This paper introduces EBSD for phase identification and illustrates the technique with examples from metal joining and particle analysis. Reduced unit cell determination from EBSD patterns is then discussed.

\section{INTRODUCTION}

The identification of unknown micrometer-sized phases in the scanning electron microscope (SEM) has been limited by a lack of a robust and simple way to obtain crystallographic information about the unknown while observing the microstructrure or morphology of the specimen. Electron backscatter diffraction (EBSD) in the SEM has become an established technique for the determination of the orientation of individual crystallites and has recently been developed into a tool that can provide identification of unknown crystalline phases.[1] Previous to the development of EBSD for phase identification, the only applicable technique for the identification of micrometer-sized phases was electron diffraction in the transmission electron microscope (TEM). Selected area electron diffraction (SAED) can provide information from submicrometer sized phases, but requires the preparation of electron transparent samples which can be very difficult and time intensive. Diffraction in the TEM is better suited to the identification of smaller sample areas due to the limited electron transparent areas of thin samples, while phase identification using EBSD is best suited to larger ( bigger than 0.1 micrometers) sample areas due to the spatial resolution of the technique. Thus, phase identification using EBSD is a complimentary technique to SAED in the TEM.[2]

Previous attempts at using EBSD for phase identification were based on the recognition of symmetry elements in the patterns. When all of the symmetry elements for a given crystal were determined, the point group of the crystal could be determined. It was found that EBSD could identify 27 of the 32 possible point groups. This approach was quite difficult and required a very good understanding of crystallography. Also, high quality patterns were required and these were obtained by using photographic film in the SEM.[3,4]

This paper will discuss the progress that has been made in using EBSD patterns in the SEM for the identification of unknown phases. The use of a large crystallographic 


\section{DISCLAIMER}

This report was prepared as an account of work sponsored by an agency of the United States Government. Neither the United States Government nor any agency thereof, nor any of their employees, make any warranty, express or implied, or assumes any legal liability or responsibility for the accuracy, completeness, or usefulness of any information, apparatus, product, or process disclosed, or represents that its use would not infringe privately owned rights. Reference herein to any specific commercial product, process, or service by trade name, trademark, manufacturer, or otherwise does not necessarily constitute or imply its endorsement, recommendation, or favoring by the United States Government or any agency thereof. The views and opinions of authors expressed herein do not necessarily state or reflect those of the United States Government or any agency thereof. 


\section{DISCLAIMER}

Portions of this document may be illegible in electronic image products. Images are produced from the best available original document. 
database in conjunction with the chemistry of the sample has now become a standard and relatively simple technique for phase identification. Recent work has shown that it is possible to determine reduced unit cells from the EBSD patterns with no a priori information about the sample. Examples of phase identification of bulk materials and particulate materials will be discussed. Finally, the determination of reduced unit cells from EBSD patterns will be discussed.

\section{EXPERIMENTAL}

In order to overcome the disadvantages of using photographic film a charge coupled device (CCD) based camera was developed. This camera consists of a single crystal yttrium aluminum garnet (YAG) phosphor that was fiber optically coupled to a cooled $1024 \times 1024$ slow-scan scientific-grade CCD: The fiber optic used was a 2.5:1 tapered optic. For most applications the CCD was binned to $512 \times 512$ resulting in exposure times of 1 to 10 seconds. The camera has been described in detail previously.[1]

The CCD camera was installed on a JEOL 6400 SEM. The specimens were mounted on a pre-tilted specimen holder at $70.5^{\circ}$ from the horizontal. The exact sample tilt is not critical as we are not measuring the sample orientation. EBSD patterns were usually obtained with an accelerating voltage of $20 \mathrm{kV}$. Sample preparation varied depending on the sample. Polished sections of metals and ceramics were prepared using standard metallographic techniques followed by a light etch to remove any deformed surface layers. EBSD patterns were obtained from many samples with little or no specimen preparation. Single crystal samples of minerals were mounted in the SEM with a crystal facet oriented toward the EBSD camera. The only specimen preparation required was cleaning with a suitable solvent. An example of a typical pattern is shown in Figure 1. Flat fielding is the only image processing

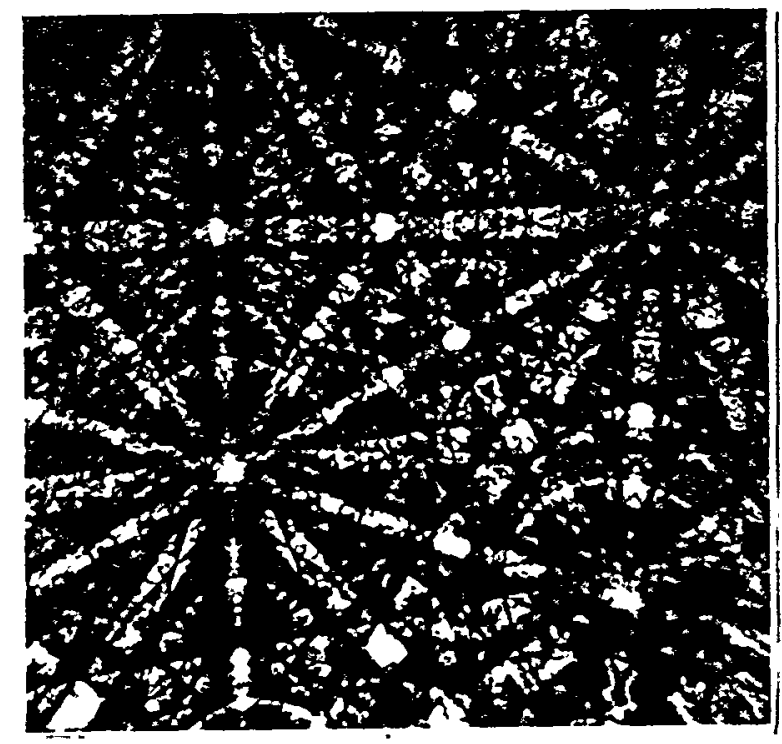

Figure 1. EBSD pattern obtained from a $5 \mu \mathrm{m} \mathrm{RuO} 2$ crystal at $20 \mathrm{kV}$. 
procedure that is normally required for phase identification. The raw image acquired with a focussed electron probe contains the crystallographic information from the specimen and artifacts related to the backscattered electron distribution and the camera. A second image, . called the flat field image, is obtained while the electron beam is scanned over a large number of grains. This effectively removes the crystallographic information from the pattern, but preserves the image artifacts. The final high quality pattern is then obtained by dividing or normalizing the raw pattern by the flat field image. Large single crystals present a problem as it is difficult to obtain a flat field image that contains no crystallographic information. In these cases it is useful to have a fine grained sample of nearly the same average atomic number as the unknown. It is then possible to obtain a flat field from the fine grained material and use it for flat fielding.[1]

\section{RESULTS AND DISCUSSION}

\section{Phase Identification Procedure}

The identification of unknown phases is accomplished in the following manner. First an EBSD pattern is obtained from the region of interest. The qualitative chemistry of the area must also be determined using either energy dispersive spectrometry (EDS) or wavelength dispersive spectrometry (WDS). This is important as the chemistry of the sample is one of the parameters used to search the crystallographic database.

The EBSD pattern is then analyzed. The Kikuchi line pairs are identified through the use of the Hough transform. The Hough transform simply transforms lines in real space to points in Hough space that can be located automatically. Once the positions of the Kikuchi lines are identified, the widths of the lines are determined. The width of the Kikuchi line pairs are inversely proportional to the spacing of the atomic planes. Once the plane spacing for a number of lines in the pattern are determined, a reduced unit cell volume is calculated. In most cases a subcell volume is obtained. The reduced unit cell volume along with the sample chemistry is then used to search a crystallographic database. We have used the Powder Diffraction File (PDF) produced by the International Center for Diffraction Data (ICDD). This database currently contains over 100,000 inorganic compounds. The typical search that includes both the unit cell volume and the chemistry requires a few seconds and will usually return at most 20 possible matches. It is then necessary to index the pattern using the database information. If a consistent set of indices for the Kikuchi lines is found, the pattern is then simulated using the database crystallographic structure. A comparison of the simulation and the experimental pattern is usually sufficient to determine the identification of the phase.[5] 


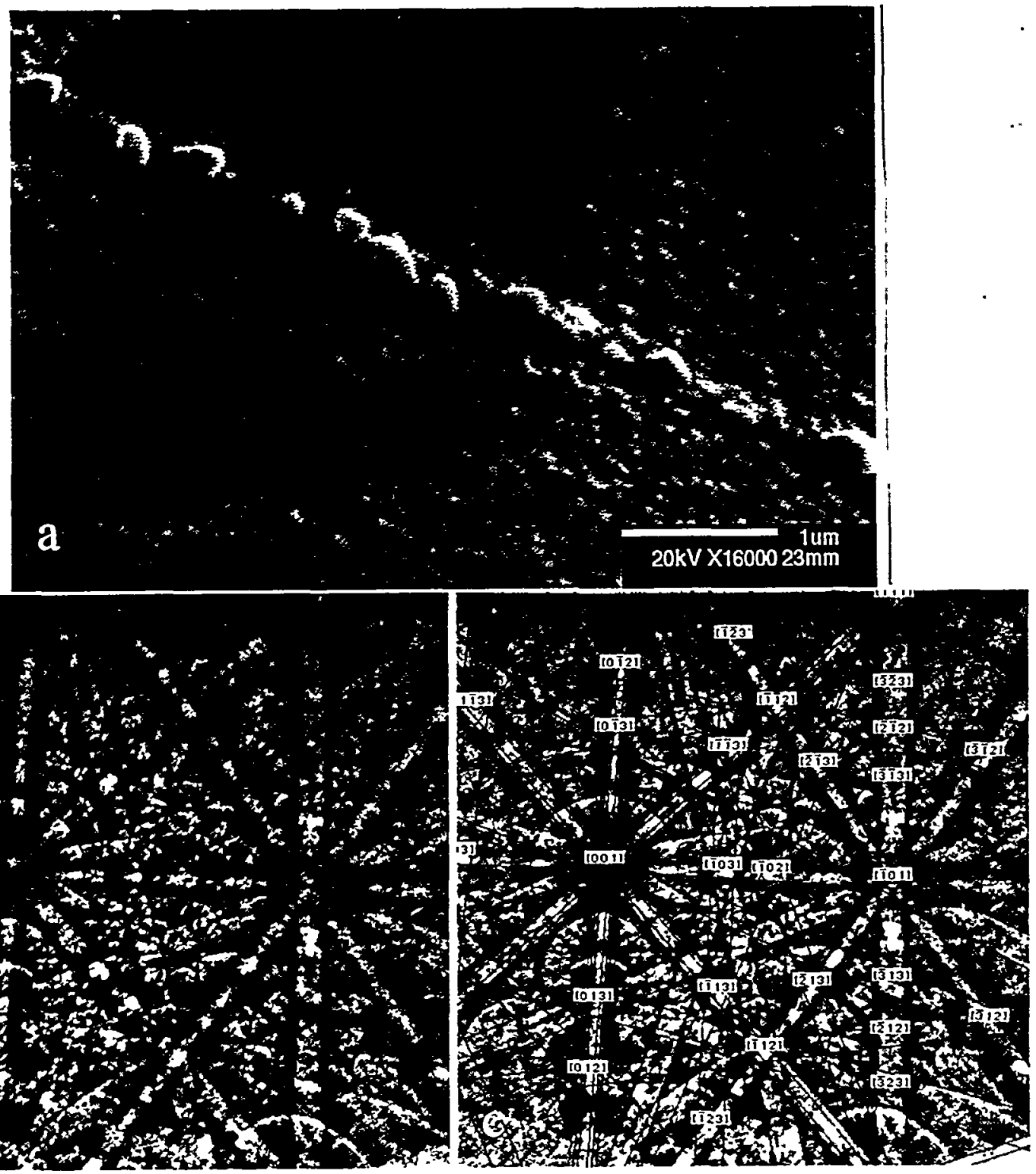

Figure 2. Identification of precipitates found at grain boundaries in a welded Ta alloy. a) SE M image of a grain boundary, b) EBSD pattern acquired from precipitate phase, c)EBSD pattern indexed as $\mathrm{HfO}_{2}$.

\section{Examples of Phase Identification Studies}

Application of EBSD Phase Identification to Welding Research

The physical properties of welds are often dependent on the phases that form during the welding process. Often these phases can be quite small and have been traditionally identified by SAED in the TEM. Figure 2 shows a grain boundary in a Ta alloy weld. The grain boundary is decorated with small precipitates (about 0.2 micrometers in size) that were shown to contain mostly Hf. Figure $2 b$ is an EBSD pattern obtained from one of the $\mathrm{Hf}$ 


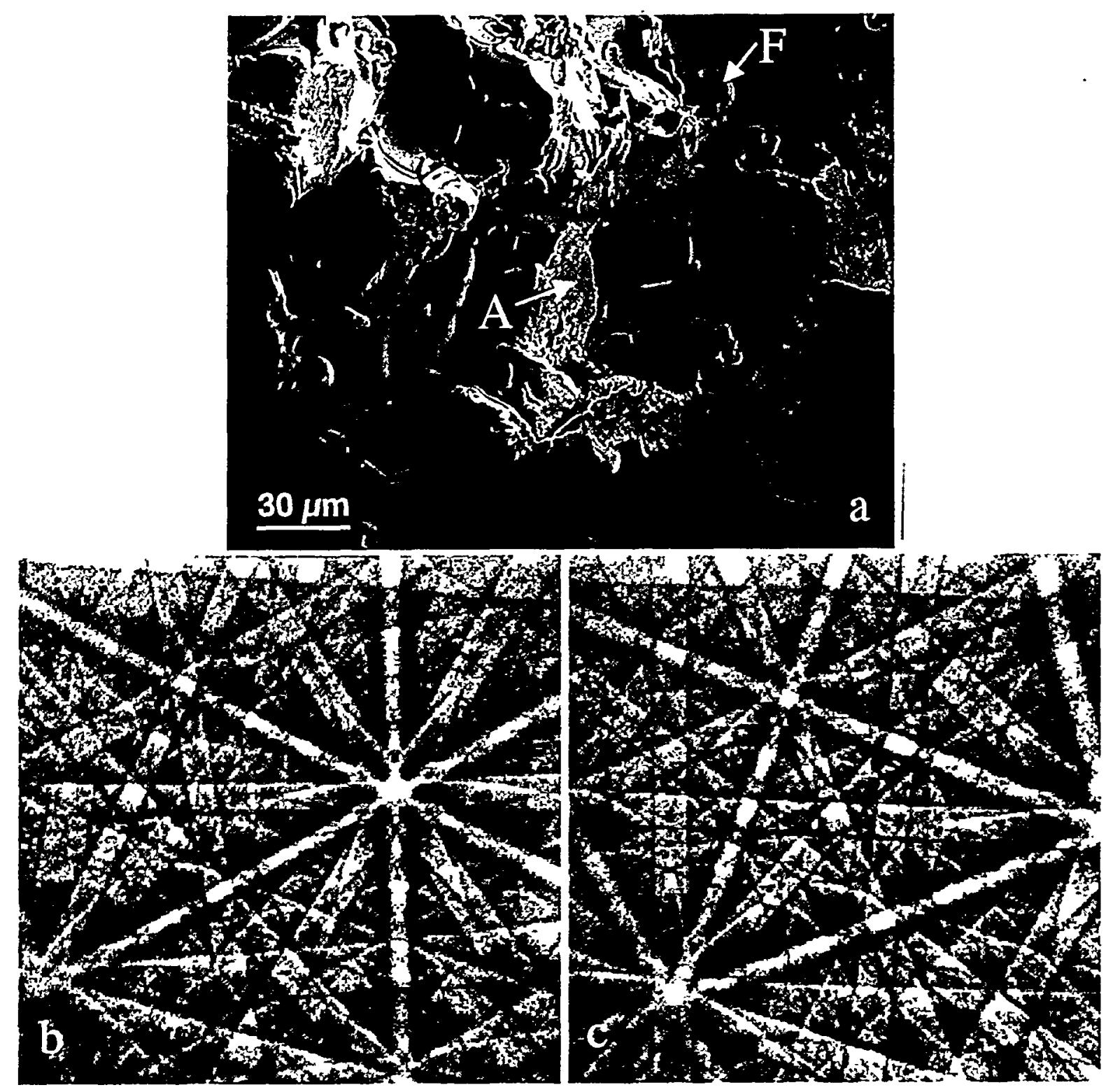

Figure 3. Identification of austenite and ferrite on a fracture surface in a welded austenitic alloy. a) SEM image of the fracture surface, b) EBSD pattern from area labeled $F$ in $3 a$, c)EBSD pattern from area labeled $A$ in $3 a$.

precipitates. The pattern was analyzed and the database searched using the calculated reduced unit cell volume and the possible chemistry of $\mathrm{Hf}$ and $\mathrm{C}, \mathrm{N}$ or $\mathrm{O}$. This search of the database resulted in 5 possible matches. The only match that could index the EBSD pattern was monoclinic $\mathrm{HfO}_{2}$. The experimental pattern overlaid with the simulation is shown in Figure $2 c$. There is excellent agreement between the experimental pattern and the simulation. The presence of $\mathrm{HFO}_{2}$ in the weld region indicates that during the welding process there was inadequate shielding of the weld resulting in the formation of the oxides. 


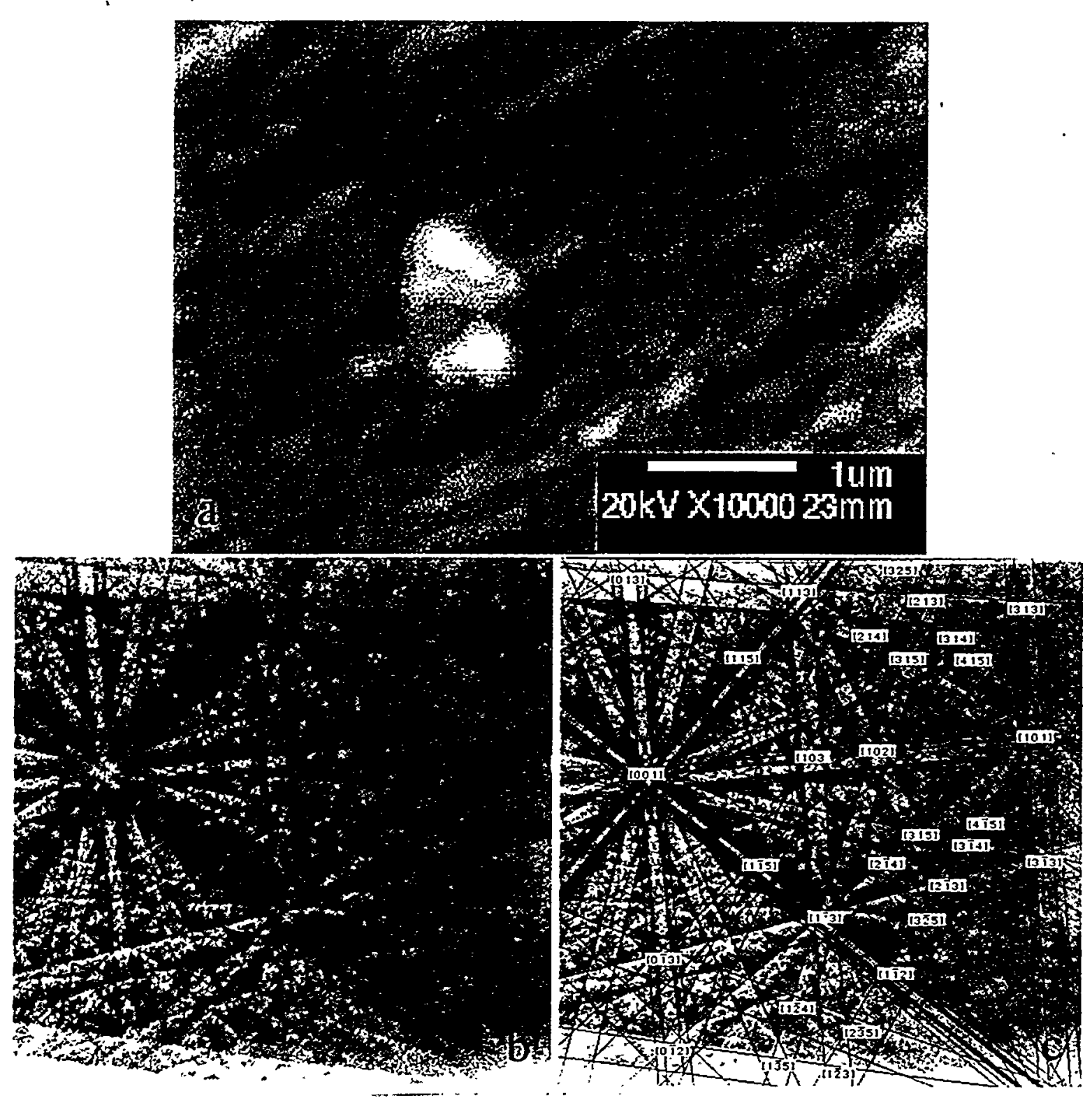

Figure 4. Identification of individual $\mathrm{Pb}$-containing particles on a carbon substrate. a) SEM image of $0.5 \mu \mathrm{m}$ particle, b) EBSD pattern obtained at $20 \mathrm{kV}$. c) EBSD pattern indexed as $\mathrm{PbO}_{2}$ ( orthorhombic).

EBSD studies need not be conducted on flat polished samples. Figure $3 \mathrm{a}$ is an example of a hot crack that formed during the welding of an austenitic alloy. There are two phases present on the fracture surface and these are indicated by arrows. EBSD patterns were obtained from the two phases. The chemistry of the regions were very similar in that they both contained Fe, $\mathrm{Ni}$ and $\mathrm{Cr}$. The EBSD patterns shown in Figure $3 \mathrm{~b}$ and $3 \mathrm{c}$ were analyzed and used to identify the globular phases at the grain boundaries as ferrite and the remainder of the fracture surface is austenite.[6] 
The identity of small particles on substrates is very difficult to determine using SEM imaging and $\mathrm{x}$-ray microanalysis. X-ray microanalysis of particles is very difficult as a result of the small particle size and the uneven surfaces of the particle. The small size results in the electron beam interacting with the substrate as well as the particle and the uneven or rough surfaces make quantitative $\mathrm{X}$-ray microanalysis very difficult if not impossible. EBSD is a useful technique for the identification of particulate materials because patterns may be obtained from individual particles. Figure $4 \mathrm{a}$ is an SEM micrograph of a small particle collected on carbon tape. $\mathrm{X}$-ray analysis showed the particle to contain $\mathrm{Pb}$ and possibly $\mathrm{O}$.

Figure $4 \mathrm{~b}$ is the EBSD pattern obtained from the particle shown in $4 \mathrm{a}$. There are at least 4 known lead oxide compounds. The phase identification algorithm identified this particle as $\mathrm{PbO}_{2}$ an orthorhombic phase. Figure $4 \mathrm{c}$ is the experimental pattern overlaid with the simulation based on the database information for $\mathrm{PbO}_{2}$. The agreement is quite good indicating that the particle has been identified as orthorhombic $\mathrm{PbO}_{2}$.[7]

\section{Structure Determination From EBSD Patterns}

EBSD patterns are formed by the elastic scattering of inelastically scattered electrons. The patterns appear as though the electrons that contribute to the pattern diverge from a point source of radiation within the sample and are therefore termed divergent beam diffraction patterns. EBSD patters are related by reciprocity to channeling patterns or rocking beam patterns. Many of the features observed in EBSD patterns are also observed in convergent beam diffraction (CBED) patterns in the TEM, although there is only an approximate

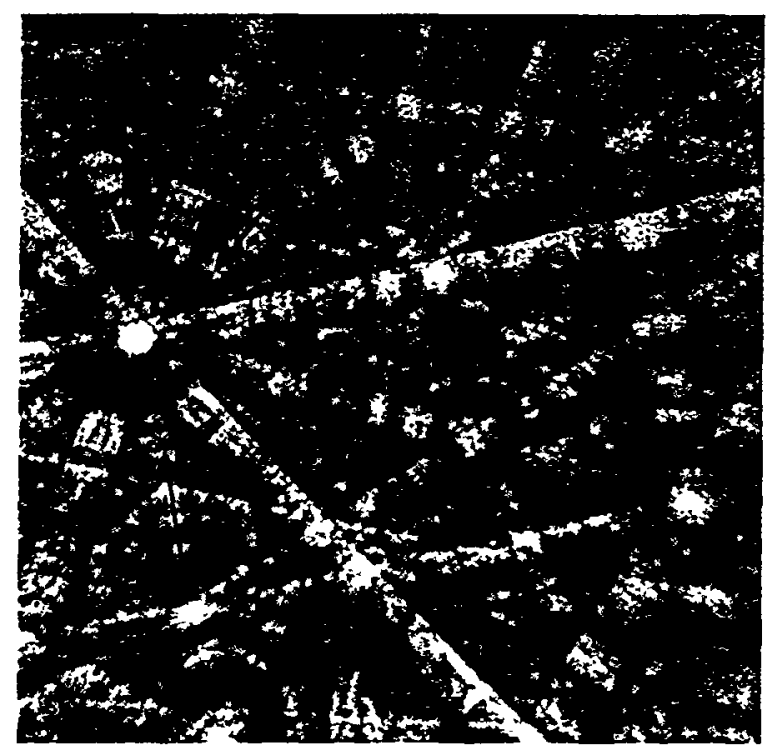

Figure 5. EBSD pattern from a $\mathrm{Cr}_{7} \mathrm{C}_{3}$ precipitate in a ferritic stainless steel. A prominent $\mathrm{HOLZ}$ ring is indicated by the arrows. 

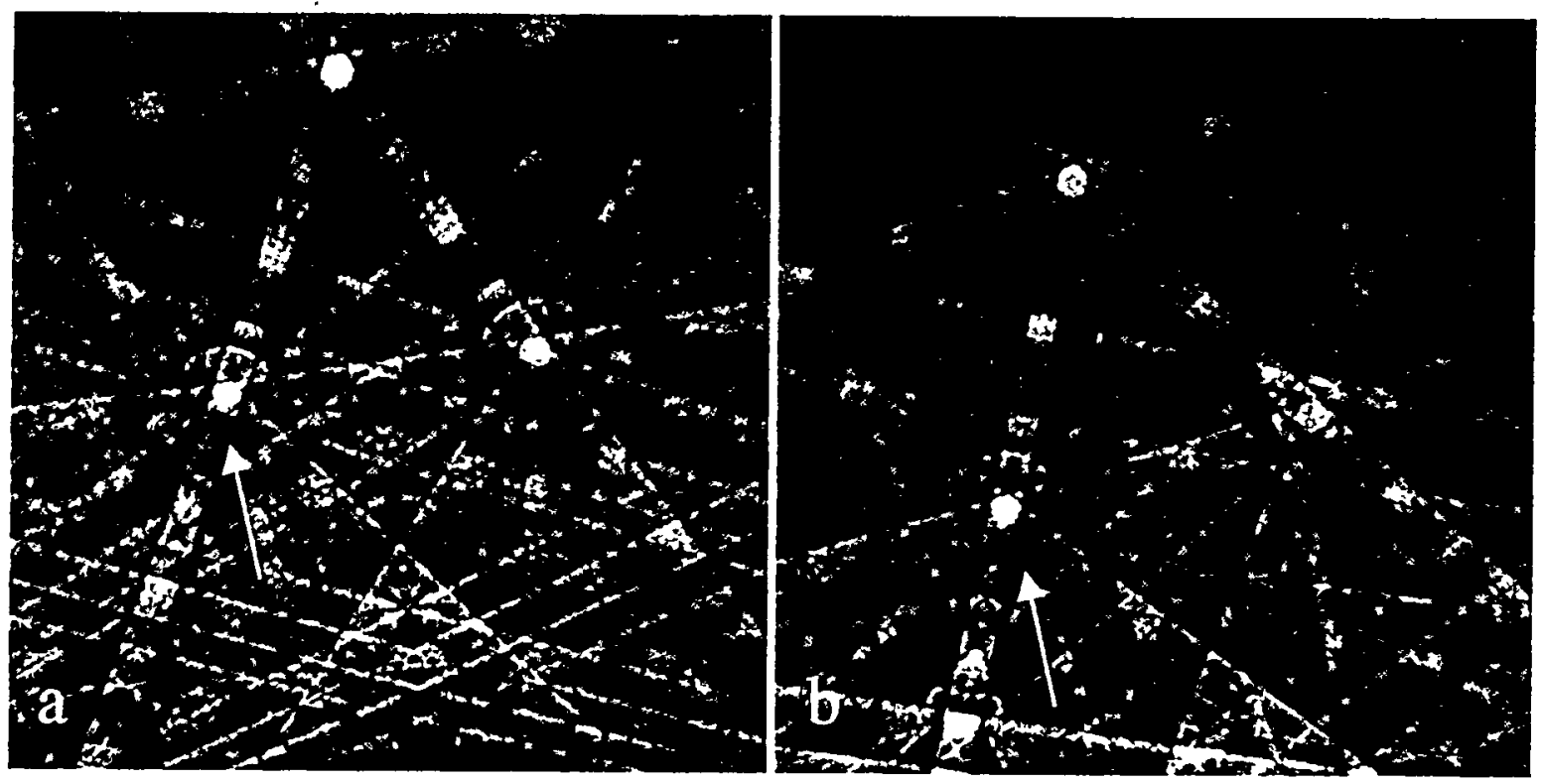

Figure 6. EBSD patterns obtained from two polytypes of SiC. The HOLZ rings analyzed are indicated by arrows. a) EBSD pattern from $6 \mathrm{H}$ polytype. b) EBSD patten from $15 \mathrm{R}$ polytype.

relationship between EBSD patterns and CBED patterns because the geometry is different in the two cases. In many EBSD patterns there are rings visible around zone axes. These rings have been shown recently to be higher order Laue zone (HOLZ) rings. These HOLZ rings correspond to an envelope of excess lines paired with deficiency lines excited close to the zone axis, but where the reciprocal lattice point is not in the zero layer.[8] HOLZ rings have been used in CBED to determine the reciprocal lattice layer spacing. The accuracy is no better than $1-2 \%$ due to lens distortions introduced by the use of short camera lengths. Recent work has shown that HOLZ rings in EBSD patterns can be analyzed in the exact same way as in CBED patterns. The only modification is that the diameter of the rings are most easily measured in terms of angle. [8]

Figure 5 is an EBSD pattern from a $\mathrm{Cr}_{7} \mathrm{C}_{3}$ carbide in a steel alloy. The [001] HOLZ ring is indicated by the arrows. The measurement of this HOLZ ring results in a real lattice spacing of $0.453 \mathrm{~nm}$ in the [001] direction that compares well with the value calculated from the crystal structure of $0.453 \mathrm{~nm}$. The accuracy of spacings determined from HOLZ rings is much greater than measurements made from the widths of the Kikuchi line pairs. This technique has been demonstrated in a large number of materials and all crystal systems with an accuracy of 0.1 to $2 \%$. The only problem occurs for higher atomic number materials where the accuracy of the technique is rather poor. This is most probably due to the rather short extinction distances in higher atomic number materials. An extrapolation technique has been developed to correct for this problem.[8]

One use of HOLZ rings in EBSD is to discriminate different polytypes. Figure 6a and $b$ are EBSD patterns obtained from two polytypes of SiC. Note the HOLZ rings indicated by the arrows in Figures $6 a$ and $b$. The measured value for the HOLZ ring indicated in figure 
$6 \mathrm{a}$ is $1.858 \mathrm{~nm}$ which is excellent agreement with the expected value of $1.846 \mathrm{~nm}$ for this zone in the $6 \mathrm{H}$ polytype of $\mathrm{SiC}$. The HOLZ ring indicated in Figure $6 \mathrm{~b}$ results in a calculated spacing of $1.545 \mathrm{~nm}$ which is in excellent agreement with the expected value of $1.538 \mathrm{~nm}$ for this zone in the $15 \mathrm{R}$ polytype. This example demonstrates that the HOLZ rings in EBSD patterns may be useful for identifying polytypes.[8]

The spacings determined from the HOLZ rings have other uses. If the spacings from three non-coplanar xone axes are measured it is possible to calculate the primitive unit cell for the specimen. The cell reduction algorithms are based on the fact that any three non-coplanar prime lattice vectors will produce an arbitrary unit cell that in a unique way represents the Bravais lattice in which it is embedded. Every Bravais lattice contains an infinite number

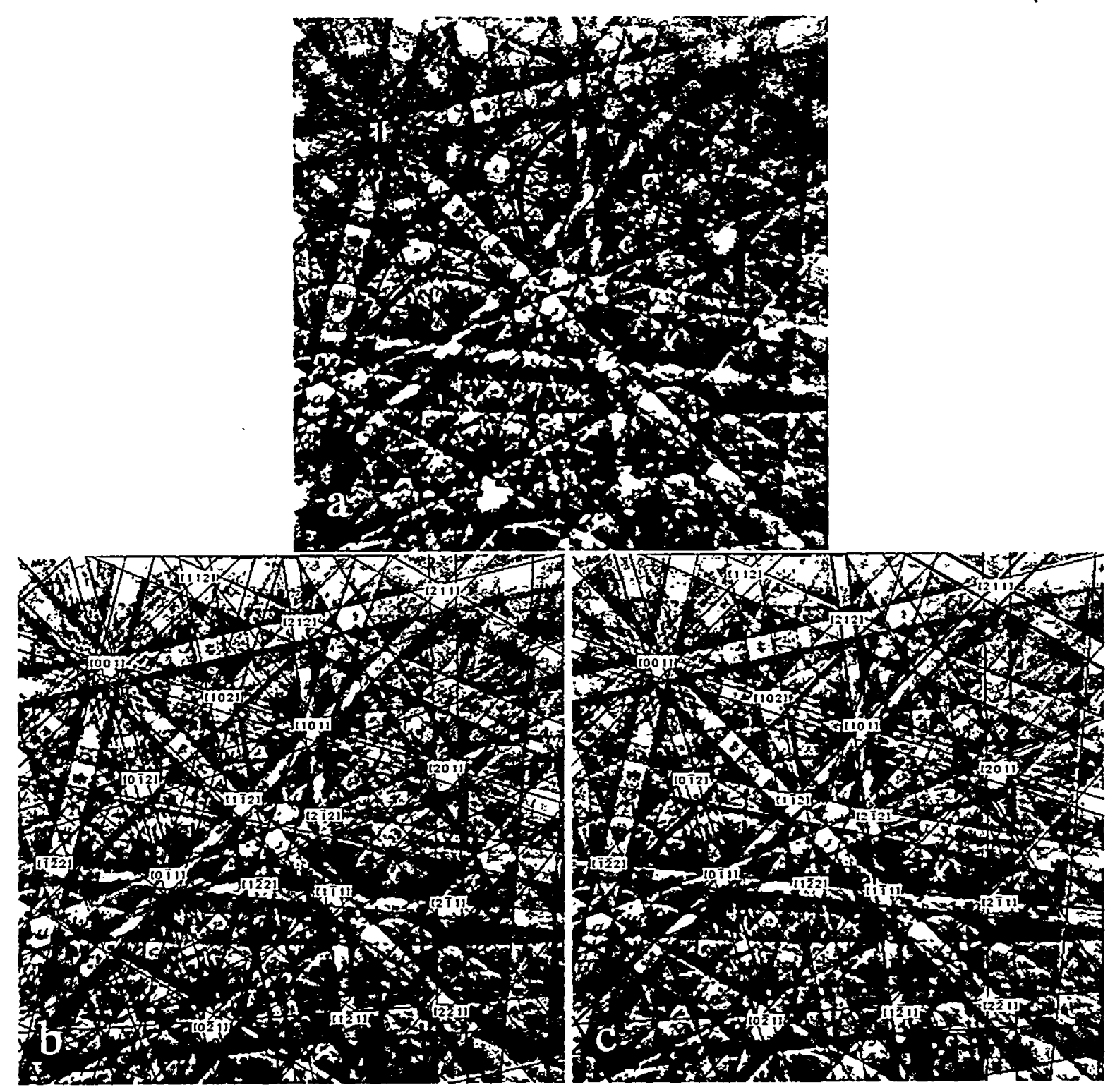

Figure 7. EBSD pattern from $\mathrm{Mo}_{2} \mathrm{C}$ precipitate in a cast iron. a) EBSD pattern indexed with primitive unit cell calculated directly from the pattern. b) EBSD pattern indexed with information from the crystallographic database. 
of primitive cells that are different in shape and size, but are equivalent in generating the Bravais lattice. Therefore, from three interatomic plane spacing measurements and the angles between the planes an arbitrary unit cell can be determined. This arbitrary primitive cell is then used as input to the primitive unit cell algorithm for the determination of the primitive unit cell of the phase. This approach has been demonstrated previously where primitive unit cells were generated from unindexed CBED patterns in the TEM.[9]

From EBSD patterns arbitrary primitive unit cells can also be constructed by combining three lattice vectors determined from the Kikuchi line pairs. However, the accuracy of plane spacings determined from the Kikuchi line pairs is relatively poor, whereas reciprocal lattice layer spacings determined by HOLZ ring analysis are much more accurate as shown above. The use of HOLZ ring measurements results in sufficient lattice spacing accuracy to determine primitive unit cells and has been demonstrated in a number of crystals.

Figure 7a is an EBSD pattern from a $\mathrm{Mo}_{2} \mathrm{C}$ precipitate in a cast iron. An arbitrary primitive unit cell was determined from the numbered zones on the pattern. The real lattice spacings determined were: zone $1,0.48 \mathrm{~nm}$, zone $2,0.56 \mathrm{~nm}$ zone $3,0.71 \mathrm{~nm}$. The angles between the perpendiculars to the HOLZ rings were measured and are: zone $1-2=32.0^{\circ}$, zone $2-3=24.3^{\circ}$ and zone $1-3=47.3^{\circ}$. These angles and layer spacings define an arbitrary primitive cell of $a=0.71 \mathrm{~nm}, b=0.56 \mathrm{~nm}, c=0.48 \mathrm{~nm}, \alpha=32.0^{\circ}, \beta=47.3^{\circ}$ and $\gamma=24.3^{\circ}$. Cell reduction is then used to determine the primitive unit cell. The resulting primitive unit cell is $\mathrm{a}=0.296 \mathrm{~nm}, \mathrm{~b}=0.298 \mathrm{~nm}, \mathrm{c}=0.48 \mathrm{~nm}, \alpha=88.2^{\circ}, \beta=89.1^{\circ}$ and $\gamma=118.7^{\circ}$ and a unit cell volume of $37.0 \AA^{3}$. This compares well with the actual cell parameters for $\mathrm{Mo}_{2} \mathrm{C}$ of $\mathrm{a}=0.3012 \mathrm{~nm}, \mathrm{~b}=0.3012 \mathrm{~nm}, \mathrm{c}=0.4735 \mathrm{~nm}, \alpha=90^{\circ}, \beta=90^{\circ}$ and $\gamma=120.0^{\circ}$ with a unit cell volume of $37.1 \AA^{3}$. Figure $7 \mathrm{~b}$ shows the experimental pattern overlaid with the simulation based on the calculated reduced unit cell and $7 \mathrm{c}$ shows the experimental pattern overlaid with the simulation calculated from the database. There is good agreement between the simulation and the experiment in both cases. Thus, it is possible to start with an unindexed EBSD pattern, calculate the primitive unit cell from the unindexed pattern and then use the calculated primitive unit cell to index the pattern. Future developments of this technique could use the calculated primitive unit cell from the EBSD pattern as an additional condition to search a suitable crystallographic database. Also, the determination of the reduced unit cell is useful when phases that are not in the database are encountered.

\section{CONCLUSIONS}

EBSD in the SEM now provides a robust and relatively simple way to identify unknown crystalline phases from their crystallography and chemistry. Procedures have been developed that permit automated identification of unknown phases in the SEM. The technique has been applied to unprepared bulk samples, polished sections, fracture surfaces and particles. Recent work shows that reduced unit cells may be directly determined from unidexed EBSD 
patterns providing another way to search crystallographic databases for matches. Phase identification in the SEM using EBSD is certainly a powerful tool for materials characterization.

\section{ACKNOWLEDGEMENTS}

This work was supported by the United States Department of Energy under contract DE-ACO4-94AL8500. Sandia is a multiprogram laboratory operated by Sandia Corporation, a Lockheed Martin Company, for the United States Department of Energy.

\section{REFERENCES}

1. R. P. Goehner and J. R. Michael, J. of Res. of the Nat. Institute of Standards and Technology, 101, 1996, pp. 301.

2. J. W. Steeds, Convergent Beam Electron Diffraction, in: Introduction to Analytical Electron Microscopy, edited by J. J. Hren, J. I. Goldstein and D. C. Joy, (Plenum Press, New York, 1979) p. 399.

3. K. Z. Baba-Kishi and D. J. Dingley, Scanning, 11,1989, p. 305.

4. K. Z. Baba-Kishi, Scanning, 20,1998, p. 117.

5. J. R. Michael, M. E. Schlienger and R. P. Goehner, Microscopy and Microanalysis, 3 Supplement, 1997, p. 879.

6. C.V. Robino, J.R. Michael, M.C. Maguire, , Welding Journal Research Supplement,77, 1998, pp. 446-s-457-s.

7. J. Small and J. Michael, Microscopy and Microanalysis, 5 Supplement 2, 1999, p. 226.

8. J. R. Michael and J. A. Eades, Ultramicroscopy, in press.

9. Y. Lepage, Microscopy Research and Technique, 21, 1992, p. 158. 